\title{
The Impact of Medicaid Managed Care on Obstetrical Care and Birth Outcomes: A Case Study
}

\author{
Ji Yan, PhD
}

\begin{abstract}
Background: As Medicaid has increasingly financed managed care plans since the 1990s, it is important to understand the corresponding impacts on the well-being of disadvantaged mothers and infants. This study examines how a Medicaid managed care (MMC) program in Pennsylvania (PA) impacts disadvantaged women's obstetrical care utilization and access as well as their birth outcomes.

Materials and Methods: This study uses a dataset of PA disadvantaged women who had multiple singleton births in 1994-2004. As to the empirical approach, we apply a linear multiple regression model to implement a pre-post design with control groups. The model also controls for unmeasured maternal birth-invariant characteristics, which affect take-up of Medicaid coverage and managed care plans.

Results: The sample for the main analysis consists of 78,346 sibling births. We find the program roll-out reduces usage of some high-tech obstetrical services and limits access to high-quality hospital services, thereby contributing to cost savings. However, implementation of the program is also associated with deterioration in birth outcomes, worse prenatal care, and an elevated risk of inappropriate gestational weight gain.

Conclusions: Cost containment through transition to MMC can be fulfilled at the price of maternal health care utilization and infant welfare. Therefore, caution is needed in design and delivery of managed care to lowincome women.
\end{abstract}

Keywords: Medicaid, managed care, obstetrical care, birth outcomes

\section{Introduction}

$\mathbf{I}_{\mathrm{s}}^{\mathrm{s}}$ N THE LATE 1980s and early 1990s, real Medicaid spending grew at an alarming average annual rate of $\sim 20 \%$. $^{1}$ An important driving force is rapid enrollment of low-income pregnant women, infants, and children following expansion of Medicaid eligibility. Frustrated with escalating costs, states initiated managed care systems in their Medicaid programs. Compared with $<10 \%$ in $1991,71 \%$ of all the Medicaid beneficiaries were in some type of managed care plans in 2010, when health maintenance organizations (HMOs) had increasingly become the typical mode. ${ }^{2,3}$ In 2011, the vast majority (77\%) of the Medicaid women were enrolled in managed care. ${ }^{4}$ Understanding the impacts of switching to Medicaid managed care (MMC) on the wellbeing of disadvantaged mothers and infants is of great interest to health professionals and policy makers.
Disadvantaged pregnant women and their infants can benefit from managed care which introduces financial incentives such as capitation to discourage inappropriate care use, improves access and continuity of care, and promotes preventive and coordinated care. However, concerns were also raised. One, health professionals can respond to the cost containment efforts in limiting provision of high-quality and medically necessary care. ${ }^{5}$ Two, managed care plans exclude the safety-net providers who had developed significant expertise to serve low-income people. ${ }^{6}$ Three, steering Medicaid childbearing women to managed care networks can lead to unmet demand for preventive care which practically causes delayed care, without a sufficient increase in obstetrical care providers. ${ }^{7}$ Four, MMC plans may offer lowcost women better care for retention and provide high-cost women with worse care to avoid them in the future. ${ }^{8}$ When the latter is the dominant story, this risk selection will

Department of Economics, Appalachian State University, Boone, North Carolina. 
adversely affect maternal and infant health. Taken together, the expected net effect of switching to MMC in a state is ambiguous.

Early studies on this topic primarily apply a cross-sectional or pre-post analysis without control groups, whereas recent research (mostly on Medicaid HMO penetration) typically uses a pre-post design with comparison groups. Overall, no consensus is reached with respect to birth outcomes. A few studies suggest beneficial effects. ${ }^{9}$ Some others report null effects on infant health. ${ }^{2,10-13}$ However, there is also evidence on adverse impacts. ${ }^{5,14}$ Likewise, previous findings are mixed on utilization of prenatal care. ${ }^{8}$ Moreover, the recent works are inconclusive regarding cesarean section, use of high-tech obstetrical procedures, maternal health and health behaviors, and hospital care. $5,6,8,12,13,15,16$

This study provides new evidence on MMC, which examines implementation of the Pennsylvania's (PA) HealthChoices program. This program implemented mandatory HMO enrollment among the Medicaid recipients. Under a 1915(b) waiver from the Health Care Financing Administration, PA introduced the program in 25 counties across three regions in 1997-2004. The first region, the HealthChoices Southeast zone was launched on February 1, 1997, which covers five counties in the Philadelphia metro area. Next, the Southwest zone initiated the program on January 1, 1999, including Pittsburgh (Allegheny County) and nine surrounding counties. ${ }^{17}$ Implementation in the Lehigh/Capital zone, which includes 10 south central counties occurred on April 1, 2002. ${ }^{18}$

Among the remaining 42 counties (used as the control groups), Medicaid beneficiaries received services through a fee-for-service (FFS) program only in 17 counties (FFS counties), or through either a voluntary capitated managed care or FFS program in the other 25 counties (Voluntary counties). In addition, the PA Medicaid-covered pregnant women up to $185 \%$ of federal poverty level throughout the study period 1994-2004. This helps us sidestep a difficult problem of isolating the effect of introducing managed care plans from the impact of changes in the Medicaid income limits.

Prior evaluation of the HealthChoices program indicates, first, its capitation payment system considerably lowers the overall reimbursements, through coordinated care and restricted access to high-quality care; second, the program actively identifies high- and low-cost users to serve the goal of tailoring care to members' level of need, which also facilitates risk selections among competing plans; third, implementation of the program, which influences take-up of Medicaid coverage and selective enrollment into plans (partly due to the enrollment exemption), is associated with fewer acute pregnancy complications; moreover, it has led to unexpectedly high demand for the HMO care providers. $16,19,20$

Since these features of the HealthChoices can influence maternal obstetrical care, access to high-quality hospital care, and birth outcomes in different directions, it is unclear what the net effect would be. To answer this question, the present study uses a unique dataset that consists of Medicaid-eligible women who resided in PA and had multiple singleton births during the years of HealthChoices expansion. The findings shed new light on whether MMC can accomplish the dual goals of reducing health care expenditure and simultaneously ensuring quality and access for low-income women.

\section{Materials and Methods}

\section{Data and study samples}

The main source of data is the PA Natality birth files covering all the birth occurrences in this state between 1994 and 2004. The birth files contain detailed information on infant health, demographic characteristics of the mother and father, characteristics of labor and delivery, mother's country of birth and county of residence, and newborn birth facility, etc. While it is infeasible to ascertain maternal Medicaid eligibility status from the data (household income or family size is not coded), we follow the literature to restrict the sample to the native-born unmarried women with no more than 12 years of education. A very high fraction of such disadvantaged women were found to be eligible for Medicaid. ${ }^{6}$

Next, we drop all the mothers residing in the South and West Philadelphia, where a pilot MMC program (known as HealthPass) was operated before 1997 but plagued by a variety of problems. ${ }^{19}$ Third, we eliminate all the mothers who were not PA residents. In the fourth step, to get a longitudinal dataset of mothers for implementing the empirical strategy below, we match consecutive singleton births to the same disadvantaged mother by mother's name, date of birth, race/ethnicity, and infant parity, under special permission of accessing restricted information in birth certificates. The final HealthChoice sample consists of 35,608 mothers with 78,346 sibling births.

Using the HealthChoice sample, we will first look at three birth outcomes: birth weight, low birth weight (LBW, birth weight $<2,500 \mathrm{~g}$ ), and preterm birth (gestational age $<37$ weeks). The next step is to investigate several channels (related to the quality or cost of obstetrical care) through which the HealthChoices program operates: prenatal care usage (onset after the first trimester, number of prenatal care visits), unhealthy gestational weight gain (excessive or inadequate weight gain: weight gain above $18 \mathrm{~kg}$ or below $6.8 \mathrm{~kg}$ for term pregnancies; or, the weekly weight gain rate above $0.5 \mathrm{~kg} /$ week or below $0.3 \mathrm{~kg} /$ week for preterm births) ${ }^{21}$ and delivery care (cesarean section, induction/stimulation of labor). Because maternal weight and height before pregnancy had not been recorded during most of the study period, we choose the above cutoffs of inappropriate weight gain which apply to any prepregnancy body mass index categories. In addition, while unhealthy weight gain is a mild and preventable health problem for the mother, it significantly increases the risk of poor birth outcomes.

Moreover, we also use information of birth facility from the birth records to introduce two additional hospital-level variables, which are publicly available on webpages of the Division of Health Informatics in the PA Department of Health. The first is presence of a neonatal intensive care unit (NICU) in the hospital for giving birth. This was the highest level of neonatal care (level 3) over the study period in the state, where the other two levels were well-newborn nursery (level 1) and special care nursery (level 2). The second is the number of staffed beds $>400$ (about the 70th percentile of the staffed bed distribution in the data). The two indicators on hospital capacity measure maternal access to high-quality and expensive hospital care as well as affect medical treatment intensity. ${ }^{5,22}$ It would be interesting to examine the exact type and amount of hospital services received by mothers, but this information is not available due to data 
limitations. Because the birth certificates had coded birth facility until 2002, we limit the sample period to 1994-2002 for the hospital care analysis and lose some useful variations of the HealthChoices expansion, especially for the Lehigh/Capital zone. The final HealthChoices-Hospital sample includes 29,251 mothers with 63,514 sibling births.

\section{Empirical approach}

The empirical model we use essentially contrasts Medicaid-eligible mothers who resided in the 25 HealthChoices counties and were exposed to the program phase-in with those in the 42 control counties who had no exposure. Specifically, we apply the ordinary least squares method to the following model, using the HealthChoices or HealthChoices-Hospital sample:

$$
\begin{aligned}
Y_{i j c t m}= & \alpha_{0}+\alpha_{1} \text { HealthChoices }_{i j c t m}+\alpha_{2} X_{i j c t m} \\
& +\alpha_{3} \text { Trend }_{c t}+\mu_{i}+\theta_{c}+\theta_{t}+\theta_{m}+\varepsilon_{i j c t m},
\end{aligned}
$$

where $Y_{i j c t m}$ is a variable on infant health, obstetrical care utilization, or access to high-quality hospital care for mother $i$ with infant $j$ born in county $c$ in year $t$ and month $m$. The indicator HealthChoices equals 1 if a mother conceived a baby after her county of residence implemented the HealthChoices program. The parameter of interest is $\alpha_{1}$, which captures the effects of this program; $X$ is a set of infant, maternal, and paternal demographic covariates that vary across births born to the same mother, including infant gender and birth order, mother's age and education, and father's race/ethnicity, age, and education. Trend is a vector of linear time trends. Below, we will estimate Equation (1) where the time trends are either zone specific (four trends in total, three of them for the HealthChoices zones and one for the control counties) or county specific.

Adoption of the HealthChoices program can alter the way of taking up Medicaid coverage among the Medicaid-eligible women in the treatment counties. Moreover, there is evidence of selective enrollment into the HealthChoices managed care plans among Medicaid beneficiaries. Aside from the observed control variables, unobserved maternal characteristics can also contribute to enrollment into Medicaid and HMO plans. We deal with this selection issue in adding mother fixed effects (FEs) $\mu_{i}$ in Equation (1), following the literature. ${ }^{5}$ In addition, Equation (1) also includes a set of maternal county of residence FEs $\theta_{c}$, infant birth year effects $\theta_{t}$, and birth month effects $\theta_{m}$. Robust standard errors are clustered at the level of maternal county of residence.

In addition to the baseline regression, Eq. 1 is used in a series of sensitivity analyses below, which consider different control counties, maternal selective migration across counties, or the women unlikely to be affected by implementation of the program. Furthermore, below we will compare results from alternative model specifications without mother FEs or time trends. Finally, we extend the analysis in two other ways: estimating an event study model and investigating potential heterogeneous effects for high-risk pregnancies.

\section{Results}

Table 1 reports summary statistics of the key variables across several mother populations. Compared with the uni- versal PA mother population (column 1), Medicaid-eligible mothers of the state (column 2) are negatively selected on multiple observed characteristics (for instance, less educated and much younger). The changes on such observed characteristics are smaller, when the sample is further restricted to women having multiple singleton births (column 3 , the working sample). In the last two columns, we find that mothers in the HealthChoices and control counties from the working sample are similar in age, education, and usage of delivery care. However, they have different racial composition, prenatal care usage, and access to high-quality hospital services. Also, women with observed differences may differ in unobserved ways. Overall, this comparison suggests it is important to properly control for observed maternal characteristics and mother FEs in the main specification: conditional on these variables, maternal residence in either the HealthChoices or control counties is determined as if randomly assigned, by which the model yields unbiased results on MMC adoption.

Table 2 presents impact estimates of the HealthChoices program, where we suppress the results of the control variables to save room. Panel 1 examines the case with zonespecific time trends in the model. The first three columns show, for the Medicaid-eligible women, the program roll-out in their counties of residence is associated with a significant decrease of $15 \mathrm{~g}$ in newborn birth weight $(p=0.025)$ plus an increased risk of LBW and prematurity by 0.7 percentage points $(p=0.063)$ and 1.5 percentage points $(p=0.001)$, respectively. The magnitude of the point estimates on adverse birth outcomes ranges from $9 \%$ to $19 \%$ (relative to the sample means). In columns (4)-(6), implementation of the HealthChoices program is found to increase the chance of late care onset by 2.4 percentage points ( $p=0.036$ ), as well as lead to a higher risk of unhealthy gestational weight gain by 1.8 percentage points $(p=0.033)$. But there is no effect on the number of prenatal care visits $(p=0.652)$.

Column (7) presents evidence on cost containment for high-tech obstetrical procedures: the estimated effect on induction/stimulation of labor is -3.7 percentage points ( $p=0.02)$. However, column (8) shows no impact on having a cesarean section $(p=0.909)$. The last two columns of Table 2 demonstrate that the HealthChoices expansion is associated with a 3.9-percentage-point decline in delivering a baby in hospitals with NICUs $(p=0.025)$ and a 6.4percentage-point decrease in having care in large hospitals $(p=0.055)$, suggesting a tendency of shifting disadvantaged women away from the high-quality hospitals (more discussion below). Panel 2 uses the specification of Eq.1 again, where zone-specific time trends are replaced by countyspecific trends. The corresponding results are very close to panel 1.

One concern about the NICU analysis is that it does not take into the account the geographic distribution of hospitals that contain NICUs. If significant changes in the geographic distribution coincide with the HealthChoices expansion, it can contribute to the estimated relationship between the treatment variable and access to hospitals with NICUs, by which the shifting effect mentioned above is exaggerated. To address this issue, we add the following time-varying variable to the model with county trends: the number of hospitals with NICUs in maternal county of residence each year. The point estimate on the HealthChoices variable shrinks to 


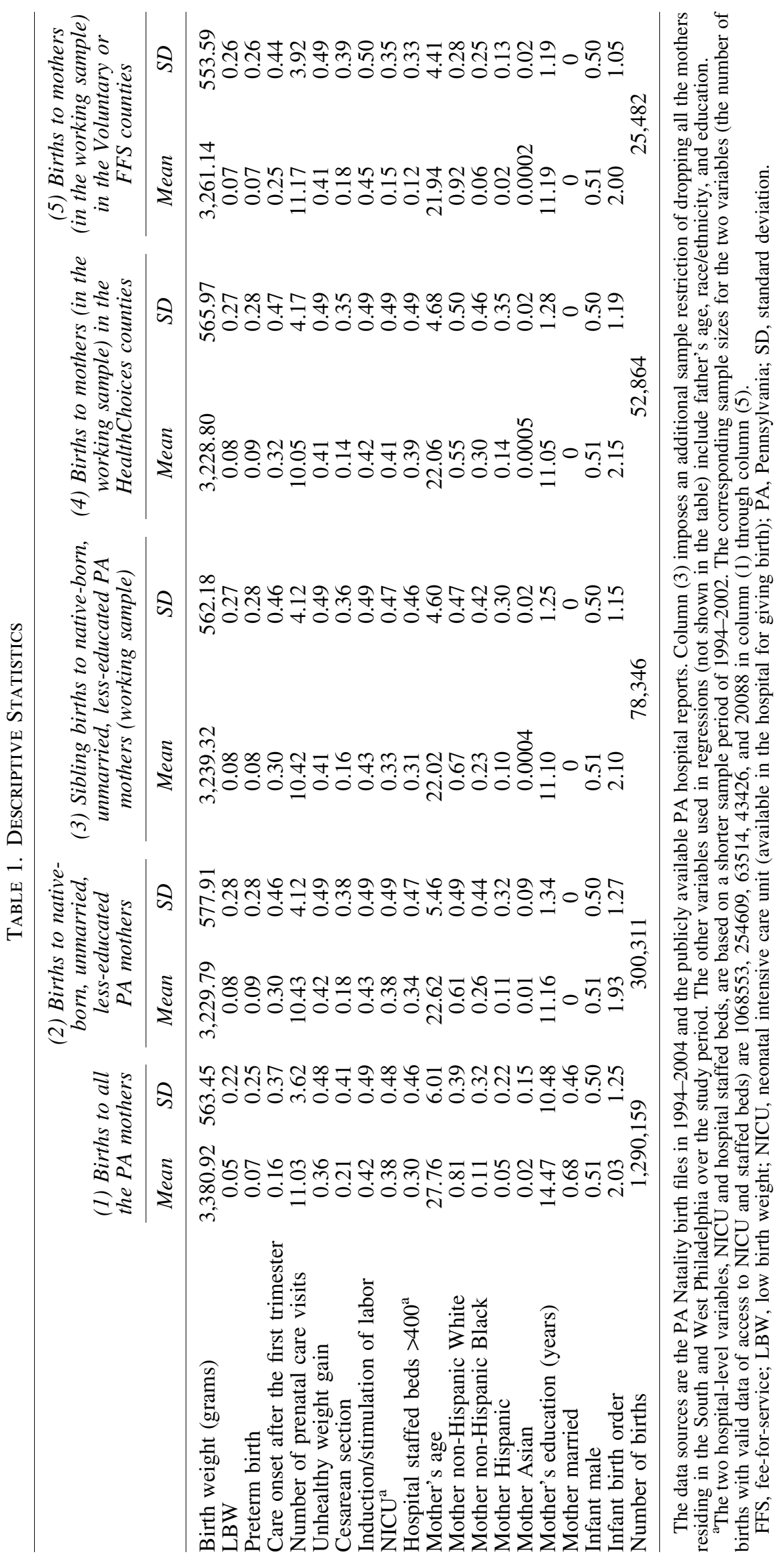




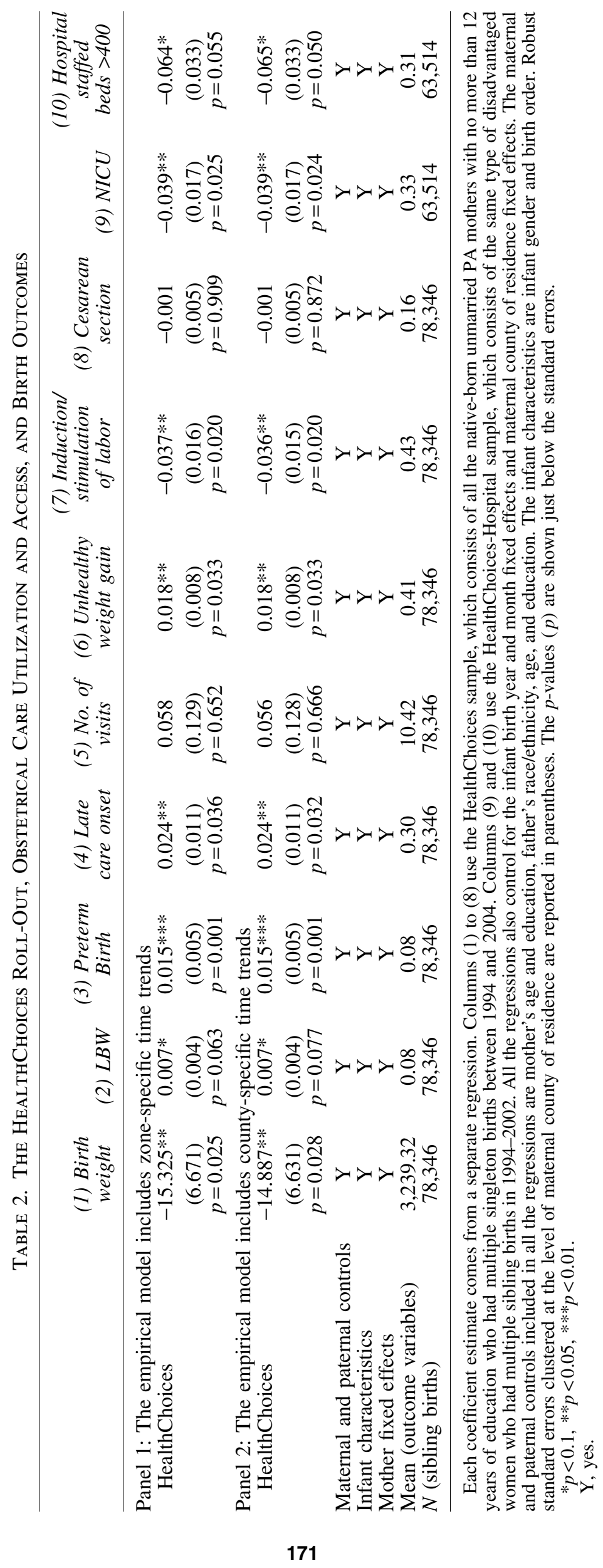


-0.028 , which is significant at $5 \%$ (Supplementary Table S1). When we additionally include the number of hospitals with NICUs across all the counties that border to maternal county of residence, the new estimate is -0.024 (significant at 10\%). Overall, for a given geographic distribution of hospitals with NICUs, we find a smaller but significant effect of moving women to lower quality hospitals (without NICUs): a 2.4- to 2.8-percentage-point decrease in having a baby in hospitals with NICUs.

Next, as a robustness check, we use only the FFS or Voluntary counties as the control counties in the model with county-specific time trends (Table 3). Consistent with Table 2, in either case, we find introducing the HealthChoices program not only negatively influences birth outcomes, prenatal care, and weight gain, but also limits usage of high-tech obstetrical care plus access to high-quality hospitals. We also perform two other sensitivity analyses (not shown for brevity). One, we examine the women who were much less likely to be eligible for welfare and affected by MMC. Specifically, we run regressions using three additional sibling birth samples of married less-educated women, unmarried more educated women, and married more educated women. None of the corresponding impact estimates appears to be significant. Two, migration of Medicaid-eligible women across the HealthChoices and control counties can be related to birth or time-variant unobserved characteristics, thereby leading to biased estimates. Adding mother FEs is not sufficient to handle this type of endogenous mobility. To address this concern, we assign women the Medicaid health delivery system (HealthChoices, Voluntary, or FFS) that they would have been exposed to had they stayed in the county where we initially observed them in the sample. Again, the results are qualitatively similar to Table 2 .

Table 4 compares results of four model specifications. Compared with the model without county time trends or mother FEs (panel 1), adding county time trends (panel 2) alters the magnitude and significance of most estimates. Alternatively, when we include mother FEs (panel 3), 6 out of the 10 estimates become larger and more precise. This is what we expected, since it controls for selection into the treatment counties and MMC plans by time-invariant maternal unobserved characteristics. When county time trends are additionally included, it does not affect the results too much (panel 4, and same results in Table 2): some estimates are different in magnitude yet statistically indistinguishable from the counterparts in panel (3).

One may wonder why the main baseline results above do not seem to be very sensitive to inclusion of county time trend, with mother FEs controlled for. One thought is that mother FEs flexibly capture how maternal unobserved characteristics drive residential sorting of disadvantaged women into the HealthChoices and control counties, whereas residential mobility may coincide with county-level population (or unemployment) change. As such, trends of countylevel factors, especially those related to population change, may be picked up to some extent (but not sufficiently) in a model with mother FEs. Moreover, it is worth mentioning that inclusion of county trends in our main specification is also in line with prior studies which use similar birth data. They show the key identifying assumption that MMC adoption is uncorrelated with various unobserved/uncontrolled time-varying county factors appears more plausible, if county trends are controlled for. ${ }^{5,8}$ One research finds, without inclusion of trends, some county-level time-varying variables (not controlled in our main model) are significantly correlated with MMC adoption and will bias the results. But this pattern no longer holds with trends in the specification. ${ }^{8}$

In another exercise, we consider an event study model, where the treatment variable of HealthChoices is replaced by a series of leads and lags. Specifically, we define the base year (the omitted category) to be the year right before implementation of the program in treatment counties, and then add six indicators of conceiving a baby 1,2 , and at least 3 years before the base year as well as 1,2, and at least 3 years after the base year. Since assigning observations of a given year in the control counties simultaneously to different leads and lags in the time window of MMC penetration (in three zones) leads to collinearity, we follow the literature to use indicators for conceptions (in the control counties) before or after the implementation window. ${ }^{8}$ Across all the outcome variables, the estimates of all the leads are insignificant, implying no significant pretreatment trends (Supplementary Table S2). The findings on the lags again suggest worse birth outcomes and reduced obstetrical care utilization or access.

Moreover, we have tried restricting the sample to the 25 HealthChoices counties and exploited variation in the timing of MMC adoption within these counties. The corresponding estimates (panel 1, Table 5) conform with the baseline findings in Table 2. Finally, it is interesting to explore the potential heterogeneous effects for high-risk pregnancies. To carry out the analysis, we create a dummy variable for a highrisk pregnancy: it equals 1 (a high-risk pregnancy) if a woman has at least one pre-existing condition (these conditions include anemia, cardiac disease, acute/chronic lung disease, diabetes, genital herpes, hemoglobinopathy, chronic hypertension, and renal disease) or at least one complication hard to prevent by medical care (such complications are malpresentation, cord prolapse, abruption placenta, and premature rupture of membrane); and it equals 0 (a normal-risk pregnancy) if she does not have any condition or complication mentioned above. ${ }^{16}$

In panel 2 of Table 5, we control for this dummy variable (results on this variable suppressed) and its interaction with the HealthChioces variable. Note the point estimate on the HealthChoices variable now captures the effect on normalrisk pregnancies. Adding this point estimate to the one on the interaction term gives the impact on high-risk pregnancies. We find, relative to normal-risk pregnancies, the adverse effects of the HealthChoices adoption on birth weight, prematurity, and access to care in large hospitals are stronger for high-risk pregnancies. Using a subsample which consists of only the mothers having high-risk pregnancies yields a similar pattern, although the estimates are less precise (not shown).

\section{Discussion}

When state Medicaid programs have increasingly financed competing, capitated managed care plans since the 1990s, there is widespread interest in assessing the corresponding impacts on health care utilization and health of the Medicaideligible population. This study examines implementation of an MMC program in PA, using mother FEs in the empirical model to deal with unobserved mother heterogeneity, which affects take-up of Medicaid and managed care plans. A prior 


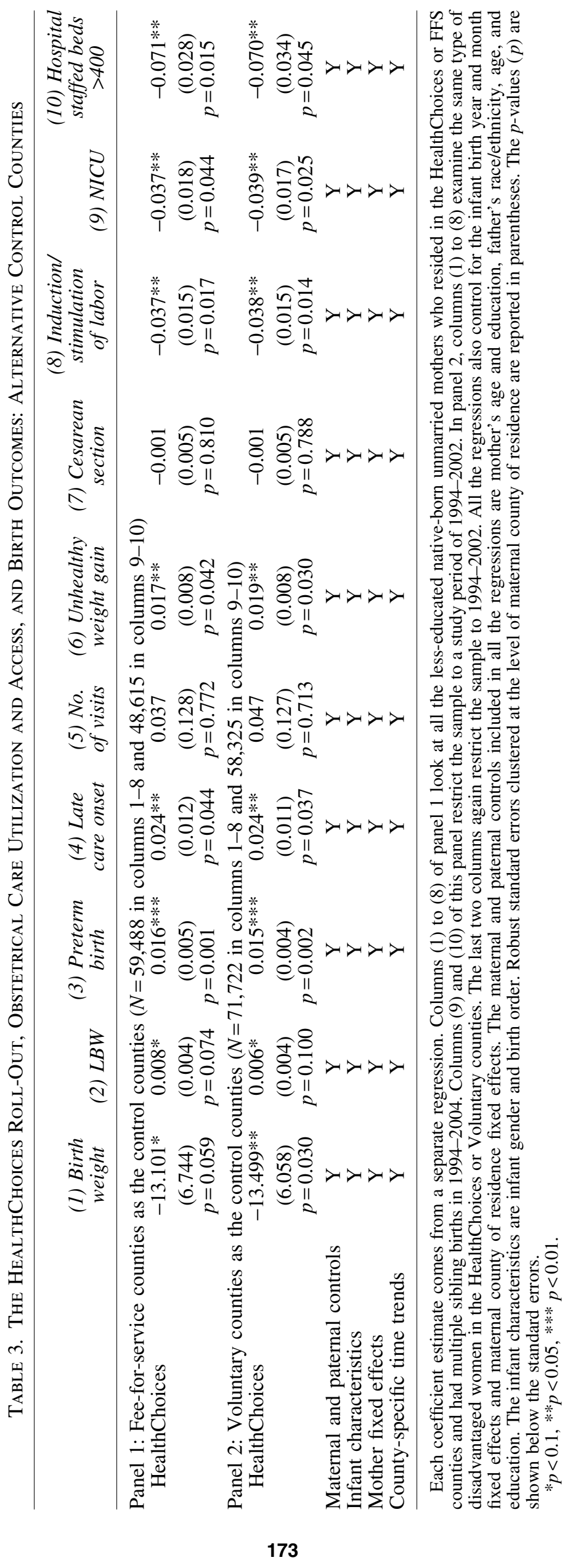




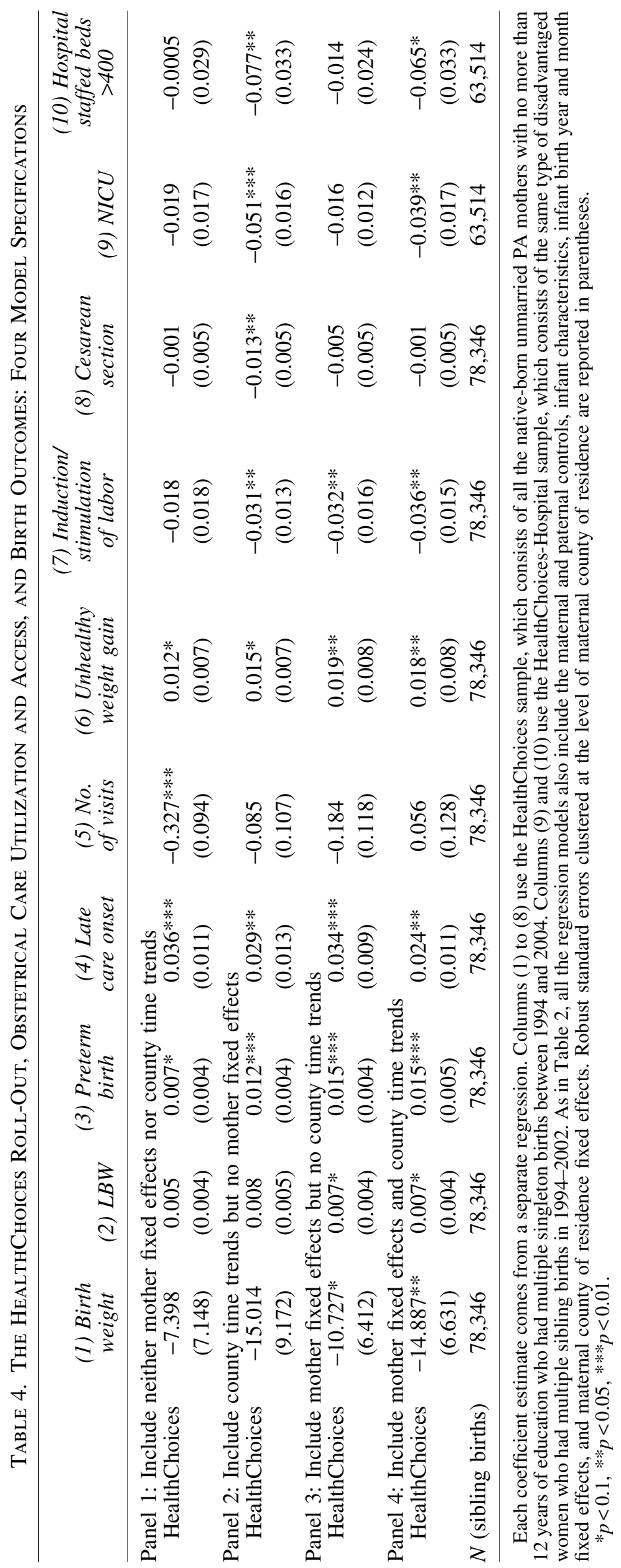




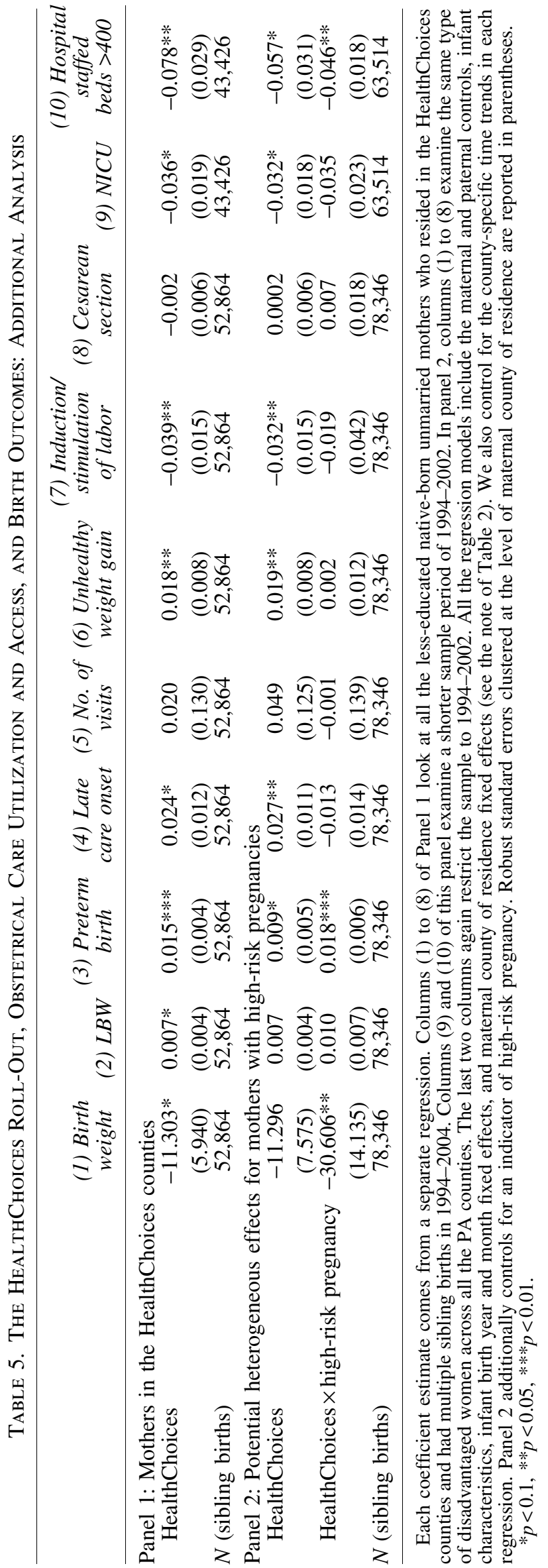

research applies the same method to find that birth outcomes deteriorated under an MMC reform during 1990s in California (CA), where most $(\sim 70 \%)$ of the Medicaid-eligible women were Hispanics or blacks. ${ }^{5}$ Interestingly, the present study reports similar findings on infant health in a state where the majority of low socioeconomic status women are nonHispanic whites. Taken together, the adverse health effects of MMC appear to be a general concern for disadvantaged populations, regardless of racial and ethnic composition.

Moreover, while the CA study shows introducing private MMC plans to Medicaid beneficiaries has no effect on induction/stimulation of labor or access to hospitals with NICUs, we find clear evidence of cost containment in PA, using the two indicators. Our study also looks at gestational weight gain, a measure not examined in the CA research. This outcome measure is closely related to the content of prenatal or coordinated care (an important aspect of care quality), which is not fully captured by the conventionally used variables of care onset or the number of care visits. It is also worth noting our finding of deterioration in the timing of prenatal care initiation is in line with several prior works on MMC. ${ }^{2,5,12}$ In addition, although the pattern of reduced usage on induction/stimulation of labor that we have reported differs from the CA study, it is consistent with another previous study which explores how an MMC program in Tennessee affects obstetrical care of high-risk women and birth outcomes. ${ }^{5,15}$

Previous research has shown that poor neonatal health (for instance, LBW and prematurity) has a lasting adverse impact on one's long-term outcomes, such as health, education, and labor force attachment. ${ }^{23}$ Given that children or infants on Medicaid are already consistently less healthy than nonMedicaid counterparts, disparities in health and other lifetime outcomes between the two populations will be widened if MMC (such as the HealthChoices program we examine in this study) undermines Medicaid children's health at birth.

A natural question arises: what policies can be put into place to minimize these concerning health impacts? As mentioned above, several potential contributors to the adverse effects may come into play during the HealthChoices expansion: incentive distortions by capitation, risk selection, unmet demand, etc. To address these issues, policymakers may consider the following strategies: one, creating sufficient incentives for private MMC plans to provide preventive care; second, promoting health education among disadvantaged childbearing women (especially the ill-informed), which will make it harder for health providers to restrict provision of high-quality but medically necessary care; three, removing regulations which restrain new obstetrical care providers from entering the market; fourth, limiting MMC plans' discretionary powers to exercise risk selection.

\section{Conclusions}

Using a unique dataset of disadvantaged women who had multiple singleton births, we find a MMC program in PA (the HealthChoices program) reduces such women's usage of some high-tech obstetrical services and limits access to highquality hospital services. However, implementation of the program is also associated with deterioration in birth outcomes, worse prenatal care, and an elevated risk of inappropriate gestational weight gain. The findings suggest that cost containment through transition to MMC can be fulfilled 
at the price of maternal health care utilization and infant wellbeing. Therefore, caution is needed in design and delivery of managed care to low-income women.

\section{Acknowledgments}

This study benefits from comments of participants of AcademyHealth Research Meeting and Association for Public Policy and Management Research Conference. The usual disclaimer applies.

\section{Author Disclosure Statement}

No competing financial interests exist.

\section{Funding Information}

No funding was received for this article.

\section{Supplementary Material}

Supplementary Table S1

Supplementary Table S2

\section{References}

1. Wade M, Berg S. Causes of Medicaid expenditure growth. Health Care Financ Rev 1995;16:11-25.

2. Sommers AS, Kenney GM, Dubay L. Implementation of mandatory Medicaid managed care in Missouri: Impacts for pregnant women. Am J Manag Care 2005;11:433-442.

3. Centers for Medicare and Medicaid Services. Medicaid managed care enrollment report: Summary statistics as of July 1, 2011. Baltimore, MD: U.S. Department of Health and Human Services, 2015.

4. Ranji U, Bair Y, Salganicoff A. Medicaid and family planning: Background and implications of the ACA. Menlo Park, CA: Kaiser Family Foundation, 2016.

5. Aizer A, Currie J, Moretti E. Does managed care hurt health? Evidence from Medicaid mothers. Rev Econ Stat 2007;89:385-399.

6. Kaestner R, Dubay L, Kenney G. Managed care and infant health: An evaluation of Medicaid in the US. Soc Sci Med 2005;60:1815-1833.

7. Griffin JF, Hogan JW, Buechner JS, Leddy TM. The effect of a Medicaid managed care program on the adequacy of prenatal care utilization in Rhode Island. Am J Public Health 1999;89:497-501.

8. Kuziemko I, Meckel K, Rossin-Slater M. Does managed care widen infant health disparities? Evidence from Texas Medicaid. Am Econ J Econ Policy 2018;10:255-283.

9. Oleske DM, Branca ML, Schmidt JB, Ferguson R, Linn ES. A comparison of capitated and fee-for-service Medicaid reimbursement methods on pregnancy outcomes. Health Serv Res 1998;33:55-73.
10. Carey TS, Weis K, Homer C. Prepaid versus traditional Medicaid plans: Lack of effect on pregnancy outcomes and prenatal care. Health Serv Res 1991;26:165-181.

11. Levinson A, Ullman F. Medicaid managed care and infant health. J Health Econ 1998;17:351-368.

12. Howell EM, Dubay L, Kenney G, Sommers AS. The impact of Medicaid managed care on pregnant women in Ohio: A cohort analysis. Health Serv Res 2004;39:825-846.

13. Kenney G, Sommers AS, Dubay L. Moving to mandatory Medicaid managed care in Ohio: Impacts on pregnant women and infants. Med Care 2005;43:683-690.

14. Schulman ED, Sheriff DJ, Momany ET. Primary care case management and birth outcomes in the Iowa Medicaid program. Am J Public Health 1997;87:80-84.

15. Conover CJ, Rankin PJ, Sloan FA. Effects of Tennessee Medicaid managed care on obstetrical care and birth outcomes. J Health Polit Policy Law 2001;26:1291-1324.

16. Hu T, Chou SY, Deily ME. Pregnancy outcomes for Medicaid patients in mandatory managed care: The Pennsylvania HealthChoices program. South Econ J 2015;82: 100-121.

17. Pennsylvania Department of Public Welfare. Medical assistance bulletin: HealthChoices southwest mandatory managed care program implementation schedule. Harrisburg, PA: Office of Medical Assistance Programs, 1997.

18. Pennsylvania Department of Health and Human Services. Review of the Commonwealth of Pennsylvania's Medicaid Behavioral HealthChoices program for state fiscal years ending June 30, 2001 and June 30, 2002. Harrisburg, PA: Office of Inspector General, 2003.

19. Johnston JM. A management success story: The Pennsylvania Medicaid managed care program. Albany, NY: Nelson A. Rockefeller Institute of Government, 2003.

20. Lewin Group. Comparative evaluation of Pennsylvania's HealthChoices program and fee-for-service program. Falls Church, VA: Lewin Group, 2005.

21. Institute of Medicine. Nutrition during pregnancy: Part I, weight gain; part II, nutrient supplements. Washington, DC: National Academy Press, 1990.

22. Picone GA, Sloan FA, Chou SY, Taylor Jr. DH. Does higher hospital cost imply higher quality of care? Rev Econ Stat 2003;85:51-62.

23. Oreopoulos P, Stabile M, Walld R, Roos LL. Short-, medium-, and long-term consequences of poor infant health an analysis using siblings and twins. J Hum Resour 2008;43:88-138.

Address correspondence to: Ji Yan, PhD

Department of Economics Appalachian State University PO Box 32051

Boone, NC 28608

E-mail: yanj@appstate.edu 